\title{
HUMAN MORPHOLOGIC MEASUREMENTS BY PHOTOGRAMMETRY
}

\author{
Bence Molnár, Károly Fekete, Péter Schrott \\ BME, Department of Photogrammetry and Geoinformatics \\ molnar.bence@fmt.bme.hu
}

\section{Introduction}

One of the most important parts of the human measurements is the facial measurements. A project at our department is to create a facial measurement system. There are several demands to get a high precision facial measurement system. For example, there are security systems at airports, forensic and anthropologic researches. These measurement systems make a 3D model automatically from the living human face. In our project, we choose photogrammetry to perform measurements. In addition to facial measurements the system won't be able to measure the face only, but other part of human body.

To build such a measurement system, there are several problems have to been solved. An important aspect of any close range photogrammetric system is to achieve an optimal photogrammetric network for special purpose, in this case for measurement of the human face. That includes selection of digital cameras, design of camera stations, modeling and calculation methods. This paper will present a solution for the main questions of photogrammetric network, gross error filtering and a processing method on the web.

In close range photogrammetry not photos with parallel axis are used, but ones with convergent axis. With this method more images are used at same processing, and the intersection of beams can be calculated with higher accuracy. The problems of network designing are presented based on Fekete ${ }^{1}$.

\section{Constraint of image scale}

The image scale is a decisive impact on the accuracy of point determining. The 1 . equation describes the maximum object distance, where the required accuracy is assured.

$\mathrm{d}=\frac{\overline{\sigma_{\mathrm{c}}} \sqrt{\mathrm{k}} \mathrm{c}}{\mathrm{q} \sigma}$

where $\sigma_{\mathrm{c}}$ are empiric errors of the $\mathrm{X}, \mathrm{Y}, \mathrm{Z}$ object space coordinates, $\mathrm{d}$ is object distance, $\sigma$ are mean errors of image coordinates, $\sigma_{\mathrm{a}}$ is the mean error of angle measurements, $q$ is the design factor of network, $\mathrm{k}$ are the quotient of independent measurements and number of images, $\mathrm{c}$ is the focal length.

Therefore the object distance in our project is mostly fixed; the equation is suitable to determining an ideal camera constant. It is important to select a well applicable camera for the project. The lens and the camera form a single unit; they have to be designed together, because the ratio of object distance and camera constant defines the photo scale. The selected lens is (Figure 1): N.E Technology L-SV2514MP (focal length: $25 \mathrm{~mm}, \mathrm{~F}: 1.4-\mathrm{C}$ ).

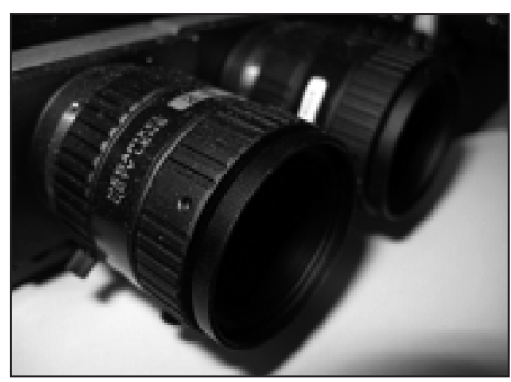

Figure 1. The selected lens 


\section{Constraint of resolution}

The minimum geometric resolution is determined by the standards for mean errors of image coordinates. The maximum is limited by financial and technical reasons. There are two disadvantages of high resolution: first, if a sequential camera is used, the amount of data will exponentially rise. The duration of the human body measurement is critical, because of the movements. The time of data gathering should be minimized. The other disadvantage is that the geometric size of the sensor is constant for technical reasons; a pixel is smaller if the resolution is higher. The charge of pixels has effect on each other; the noise will increase in higher resolution.

The critical key issue of photogrammetry is the automatic point identifying. The currently used methods for identifying are working unreliable with homogenous textured surfaces, such as in case of human face. The identifying can be improved by projecting a pattern on the surface. If more images are available with different patterns from the same object, accuracy can be improved. That means, sequenced images have to be used, therefore a high speed camera is needed.

When the camera model was selected it was an important issue that the full control of cameras has to be done by computer. The camera is

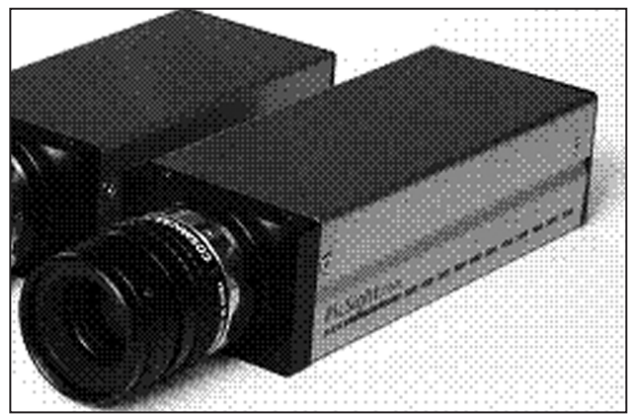

Figure 2. The selected camera programmable with an SDK (Software Development Kit), developed by the manufacturer. The selected camera is (Figure 2): PicSight GigE P202B-GigE-AR color camera (sensor size: $1 / 1.8$ " CCD, resolution: $1624 \times 1236$ pixel, speed: $14 \mathrm{fps})$.

\section{Depth of field constraint}

High accurate measurement results in a sharp image. If the projection of a point is smaller than the physical size of the pixel, the projection is sharp, otherwise a blur circle (circle of confusion) occurs. In most cases a blur circle bigger than a pixel is allowed, if the digitizing mean error is still acceptable. The depth of field (DOF) is determined (Figure 3) by the camera constant and the aperture size (f-number).

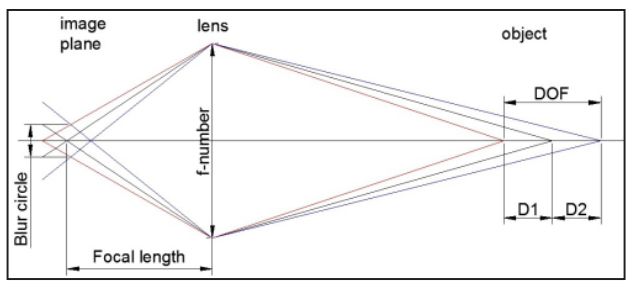

Figure 3. Depth of field

Facial measurement system requires a low object distance, compared to the relatively large size of the head. That's why the system should cope with large DOF. The object distance will be approximately $1.5 \mathrm{~m}$ and the f-number will be 5.6 to get an optimal result.

\section{Constraint of intersection and incidence angles}

The accuracy of a point identifying depends on the incident angles. Since the face is a complex surface, this constraint requires applying more cameras. In photogrammetry usually more cameras are used, typically two. In this 
case due to the complexity of the face, four cameras are needed. The accuracy of spatial measurements will be higher if the intersection angle of camera axis is the maximum that can be set. The best camera configuration is equilateral triangle-sided, square-based pyramid, on the base are the cameras, and on the peak is the object ${ }^{2}$.

\section{Constraint of lighting}

The big f-number and the sequential photography require a bright object surface. Therefore not only the camera stations but light sources should be designed too. The lighting should be homogenous at all parts of the face. The bright object can cause difficulties if a pattern is projected to the object. The projector has to be very bright, to highlight the contrasts on the object, and support the automatic point identifying.

\section{Gross errors and robust estimation}

The automatic point identifying is a critical point of the measurement system. The automatic algorithms are based on contrast difference calculation. Due to lighting and homogenous texture of skin there can be several errors during recognition. The false recognized point pairs are called gross errors in literature. If gross errors still exist in final calculation, the final accuracy will be very low. The calculation in photogrammetry usually made by Least Squares Method (LSM), which is especially sensitive to gross errors ${ }^{3}$. LSM is a very good method to minimize residuals in a fast and easy way. If the base equations of a system are linear, the LSM gives the result in one step, no other calculation and linearization is needed.

In photogrammetry beam equalization is the classical calculation method, but it uses nonlinear equations, that are not ideal for LSM.
There is an other calculation method, called Direct Linear Transformation (DLT), which gives a direct connection between image coordinates and spatial coordinates ${ }^{4}$, as shown in equation 2 and 3. As the name shows, it is based on linear equations, therefore if LSM is used, no linearization is needed.

$\mathrm{L}_{1} \mathrm{X}+\mathrm{L}_{2} \mathrm{Y}+\mathrm{L}_{3} \mathrm{Z}+\mathrm{L}_{4}-\mathrm{xL}_{9} \mathrm{X}-\mathrm{xL}_{10} \mathrm{Y}-\mathrm{xL}_{11} \mathrm{Z}-\mathrm{x}=0$

$\mathrm{L}_{5} \mathrm{X}+\mathrm{L}_{6} \mathrm{Y}+\mathrm{L}_{7} \mathrm{Z}+\mathrm{L}_{8}-\mathrm{yL}_{9} \mathrm{X}-\mathrm{yL} \mathrm{L}_{10} \mathrm{Y}-\mathrm{yL} \mathrm{L}_{11} \mathrm{Z}-\mathrm{y}=0$

where $\mathrm{x}, \mathrm{y}$ are image coordinates, $\mathrm{X}, \mathrm{Y}, \mathrm{Z}$ are spatial coordinates and $\mathrm{L}_{\mathrm{i}}$ are DLT parameters. An other advantage of DLT is that, no calibrated camera is needed. It enables to change settings on camera system, without a new calibration.

Other disadvantages of LSM are gross errors that have to been eliminated. Estimating methods that are insensitive for gross errors, named are robust estimations. Some methods make gross error filtering before estimation; other ones make it an iterative way, and continuously reweight the measurements. To make an automatic processing algorithm, the second way is a better choice.

The goal of these methods, that LSM can be used iteratively, with new weight matrices based on previous estimation residuals. The theoretical backgounds of these methods is that the clean (without gross error) measurements have normal distribution, but if gross error exits, the distribution differs ${ }^{3}$.

$\mathrm{F}=(1-\varepsilon) \Phi+\varepsilon \mathrm{H}$

where $\mathrm{F}$ is the distribution model of errors, $\Phi$ is normal distribution function, $\mathrm{H}$ is the unknown distribution of gross errors, $\varepsilon$ is the probability of gross errors. 

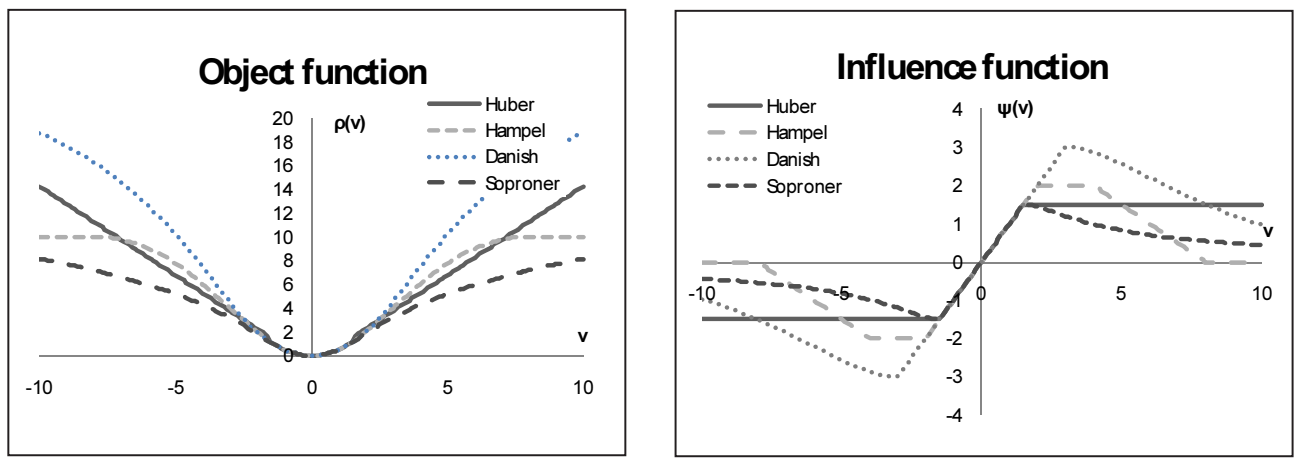

Figure 4. Density and influence function

These methods define one or more threshold, where other distribution is used in the probability density function. The first threshold always defines the part of objective function with normal distribution. The main objective functions are Huber-, Hampel-, Danish- and Soproner-functions ${ }^{5}$.

The influence function is the derivation of objective function. It shows the errors' effect on the global estimation. Influence function divided by $\mathrm{x}$ forms the weight matrix.

The objective functions are shown on the left side of Figure 4. The only one objective function which is convex is the Huber function ${ }^{6}$. (A non-convex object function has the disad- vantage that, it is not convergent. A non convergent calculation is not ideal for an automatic method, that's why Huber method is chosen for estimation.

$\psi(x)=\left\{\begin{aligned} \mathrm{x}, & |\mathrm{x}| \leq \mathrm{a} \\ \mathrm{a} \frac{\mathrm{x}}{|\mathrm{x}|}, & |\mathrm{x}|>\mathrm{a}\end{aligned}\right.$

where $\psi$ is the influence function and a is the threshold for gross errors. The value of $\mathrm{a}$ is usually 1.5 but can be changed. The estimation steps are as follows: first an initial estimation with an identity weight matrix has to be done. The following estimations are based on previously calculated errors. Iteration runs while the optimized result is calculated. At the
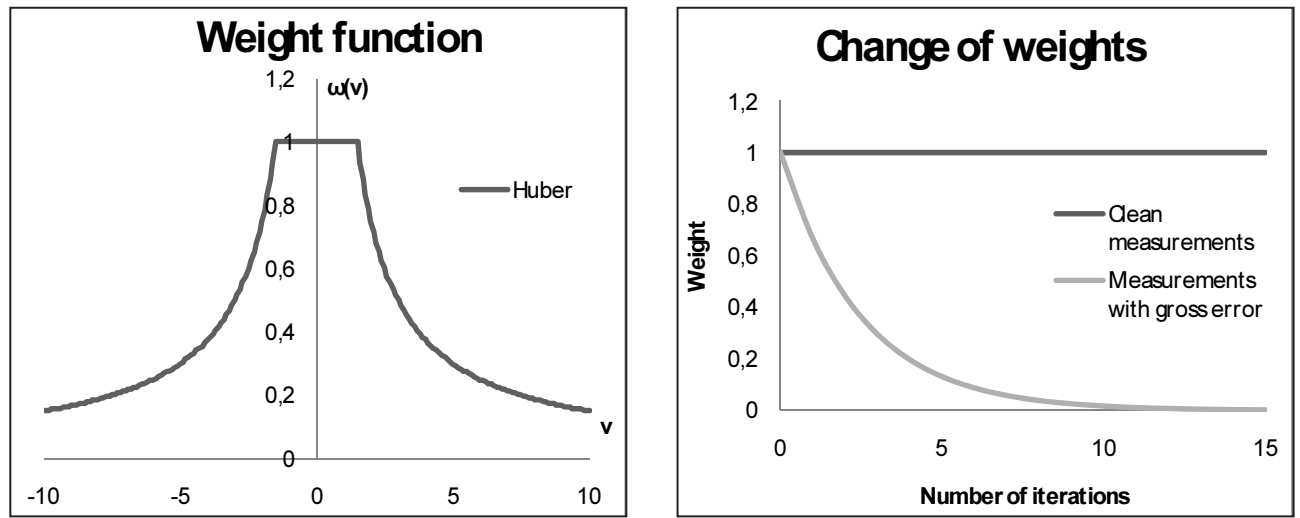

Figure 5. Weight function and weights during iterations 
end the measurements with gross errors have weights around zero, all other measurements around one (Figure 5). That means LSM can be used for estimation, the gross errors do not have effect on the final result.

To test the algorithm a web based photogrammetry application was developed. This application can be reached web, therefore available for everybody and everywhere. Own photos can be uploaded and the measurements can be done. Inexperienced users produce large amount of data that are often corrupted by gross errors. These kind of data sets are useful for testing the method and for selecting a proper threshold for the Huber method.

\section{Conclusion}

For development of a facial measurement system the best applicable equipment should be used. The best affordable cameras and lens were selected for the project. The good camera and light source configuration was defined to improve accuracy. A properly working calculation algorithm was developed for the spatial measurements, and gross errors were filtered out.

In the future a new algorithm should be developed for identifying points on the homogenous skin. Finally the photogrammetric network should be realized.

\section{REFERENCES}

1. Fekete K. Network design issues in close range photogrammetry (in Hungarian). Geodézia és Kartográfia III, 2006;12-23.

2. Mason S. Conceptual Modell of the Convergeent Multistation Network Configuration Task. Photogrammetric Record 15(86), 1995;277-299.

3. Detrekối Á. Adjustment calculation (in Hungarian), Budapest: Tankönyvkiadó, 1991.
4. Abdel-Aziz YI, Karara HM. Direct Linear Transformation from Comparator Coordinates into Object Space Coordinates ASP Symposium on Close-Range Photogrammtery, Falls Church, VA, 1971;1-18.

5. Závoti J. Robust estimation methods in geodesy, Dr Habil Thesis, 1996;1-35.

6. Huber PJ. Robust Statistics, John Wiley \& Sons, New York, 1981.

\section{Bence Molnár}

BME, Department of Photogrammetry and Geoinformatics

H-1111 Budapest, Múegyetem rkp. 3. 